\title{
INTEGRATING WIRE CHAMBERS FOR BEAM TUNING IN THE CERN SPS EXPERIMENTAL AREAS
}

\author{
P. DREESEN and G. VISMARA \\ CERN, Geneva, Switzerland
}

\begin{abstract}
Wire chambers operating in the charge integrating mode are used for beam tuning in the SPS experimental areas. They are characterized by a wide dynamic range $\left(10^{4}-10^{11}\right.$ particles/s) and can be adapted for spatial resolution between 1 and $6 \mathrm{~mm}$.

This paper describes the charge integrator, the multiplexer and the readout system. Simultaneous display of beam profiles for four chambers in both horizontal and vertical planes, with 32 channels each or one chamber up to 16 times per beam burst, are possible. An account of the system performance, after fourteen months of operation, is given.
\end{abstract}

\section{Introduction}

In the Cern SPS (Super-Proton-Synchrotron) experimental areas, the beam lines require a considerable amount of instrumentation for tuning and monitoring purposes. Among the various beam detectors an important role is played by a system of Analog Wire Chambers (AWC).

The AWC operating in the charge integrating mode has the following characteristics:

1) operation in an extremely wide range of intensities, from $10^{4}$ up to $10^{11}$ particles/s by combining the intrinsic dynamics of the associated electronics and the gain of the chamber;

2) 32 bins histograms with an adaptable resolution from 1 to $6 \mathrm{~mm}$;

3) low cost and simplicity of the associated electronic set-up;

4) fast data acquisition, with possibility of sampling beam profiles up to 16 times per beam burst (1.4 s);

5) high degree of reliability and good long term stability.

\section{Chamber}

The chambers, the main parameters of which are listed in table 1, have two orthogonal wire planes with a sensitive area of $100 \times 100 \mathrm{~mm}^{2}$ or $200 \times 200 \mathrm{~mm}^{2}$. Adapters can be used to select the chamber space resolution according to the beam size, by grouping 1, 2, 3 neighbouring wires into one electronic channel $(32,64,96$ active wires).

The choice of the operating gas is not important as far as gain is concerned, however the use of an "inorganic" mixture ( $\mathrm{A}+\mathrm{CO}_{2}$ in our case) is neces-
TABLE 1

Parameters of the multiwire proportional chambers.

\begin{tabular}{lcc}
\hline \multicolumn{1}{c}{ Sensitive area } & $100 \times 100 \mathrm{~mm}^{2}$ & $200 \times 200 \mathrm{~mm}^{2}$ \\
\hline $\begin{array}{l}\text { Sense wire spacing } \\
\begin{array}{l}\text { Sense wire diameter (gold-plat- } \\
\text { ed tungsten) }\end{array}\end{array}$ & $1 \mathrm{~mm}$ & $2 \mathrm{~mm}$ \\
$\begin{array}{l}\text { Spacing between high voltage } \\
\text { and signal plane }\end{array}$ & $5 \mu \mathrm{m}^{\mathrm{a}}$ & $20 \mu \mathrm{m}^{\mathrm{a}}$ \\
$\begin{array}{l}\text { High voltage plane - alumini- } \\
\text { um foil thickness }\end{array}$ & $\begin{array}{l}10 \mu \mathrm{m} \\
\text { Mylar, } \\
25 \mu \mathrm{m}\end{array}$ & $\begin{array}{l}\text { Aluminium } \\
\text { or Kapton, } \\
25 \mu \mathrm{mm}\end{array}$ \\
& & $25 \mu \mathrm{m}$
\end{tabular}

a On both edges of the sense plane are included three guard wires of $30,50,100 \mu \mathrm{m}$ diameter respectively.

sary to minimize aging effects due to extended beam exposure.

Most of the chambers are mounted on a vertically movable support and can be remotely extracted from the beam when not used.

\section{Electronics}

A simplified block diagram is given in fig. 1 . Each electronic channel consists of an active integrator (appendix) with a very low voltage drift $(\leq 0.5 \mathrm{mV} / \mathrm{s})$. Four channels are assembled on one pluggable card and each wire plane has 32 channels. An analogue FET multiplexer allows a fast sequential scanning of all channels ( $\leq 2 \mu \mathrm{s} / \mathrm{chan}$ nel). From the chamber location, the output signals are transmitted, through several hundred metres of twisted pair cable, to the electronics hut, and via an interface to a CAMAC station where 


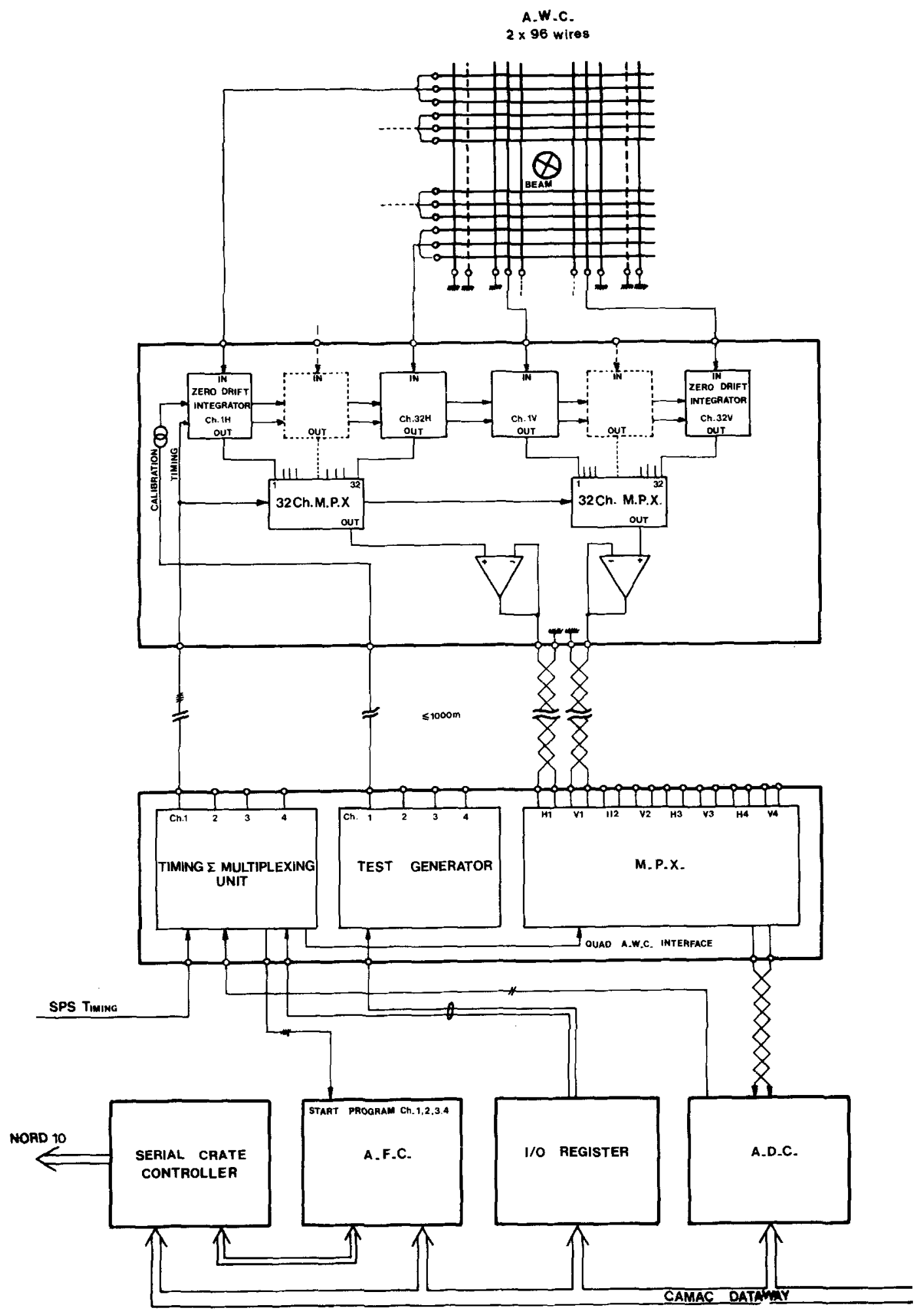

Fig. 1. Electrical block diagram of the system. 
a processing unit $(\mathrm{AFC})^{1}$ ) and an $\mathrm{ADC}$ are located.

The interface, which allows grouping up to four chambers ( 8 planes), is used to provide the timing and multiplexing signals as well as an analog observation output of a presettable channel. Furthermore, it is able, on computer request, to generate calibration signals among bursts, in order to check off-set, drift, gain and linearity of the integrators.

The AFC is a CAMAC module, developed at CERN for general application, which works as a central processor to drive the interface, the ADC and the $\mathrm{I} / \mathrm{O}$ register. It is loaded by the computer and enables the multiplexing and reading of each channel by a fast ( $20 \mu$ s conversion time) 10-bit ADC during or at the end of the burst.

The data are temporarily stored in the AFC's

S1/MWPC 3 HORIZONTAL PROFILE $=1977 \cdot 03 \cdot 25 / 10: 33$

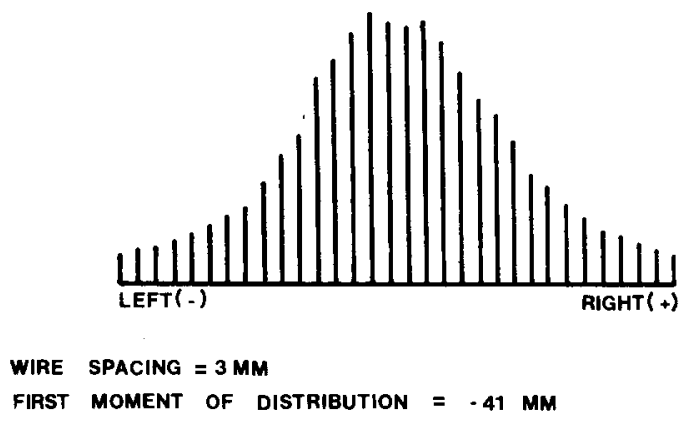

S1/MWPC 4 VERTICAL PROFILE $=1977-03-25 / 10: 36$

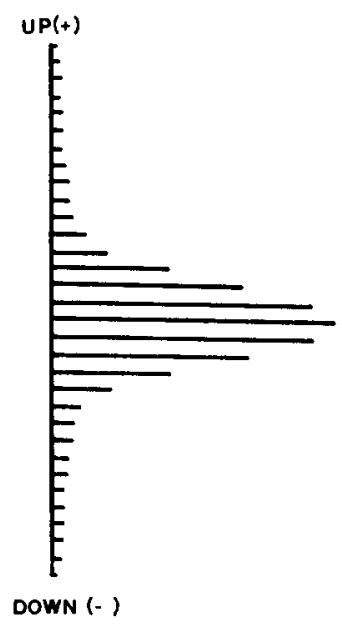

WIRE SPACING $=3 \mathrm{MM}$

FIRST MOMENT OF DISTRIBUTION $=-251 \mathrm{MM}$

Fig. 2. Typical beam profile display on Tektronix 611 . memory (1024 words) until the computer asks and treats them for display on a memory cathode-ray tube or line printer.

Some typical displays of the beam profile are shown in fig. 2 .

\section{Performances}

With a gas mixture of $\mathrm{A}+\mathrm{CO}_{2}(50 \%+50 \%)$ only negligible deposits were noticed on the wires and chambers working in the ionization region, have withstood up to $10^{17}$ particles without any apparent damage.

Accuracy among channels, on the complete electronic system, is smaller than $\pm 0.25 \%$ and linearity better than $\pm 0.15 \%$.

For a $1 \%$ accuracy no adjustments are required for a variation of 100 times in beam intensity; the dynamic range can be enlarged to $10^{6}$ by simply adjusting the high voltage. At intensity above $10^{10}$ particles/s the integrating capacitor is increased.

A negligible amount of electronics failure and practically none on the chambers, after fourteen months of operation, has shown up.

\section{Conclusions}

The AWC has proved to be a very practical and reliable instrument for beam tuning. It can be easily adapted to a wide range of intensities and beam geometries.

Five beams of the West Experimental Hall, where the intensity ranges from $10^{5}$ to $10^{11}$ particles/s, involving 10 chambers, are equipped with such a system.

An extended use of the system is foreseen for all beams of the North Experimental Areas, where 24 chambers of different geometries are now installed and ready for the beam tests.

\section{Appendix}

\section{The charge integrator}

Assuming a maximum total beam intensity of $10^{10}$ particles/s (which gives in the worst case $0.2 \times 10^{10}$ particles/channel.s) and that in a $10 \mathrm{~mm}$ gap, about 100 ion pairs/particle are produced, the integrator capacitor $\left(\mathrm{C}_{1}\right)$ to obtain a maximum output of $10 \mathrm{~V}$, with a unity chamber gain, is $3.2 \mathrm{nF}$. For higher total beam intensity $\left(\leq 3 \times 10^{11}\right) \mathrm{C}_{1}$ is increased to $100 \mathrm{nF}$.

In order to cover a dynamic range of $10^{6}$ keeping a $1 \%$ accuracy, the chamber gain has to be increased to $10^{4}$ and the output resolution of the in- 


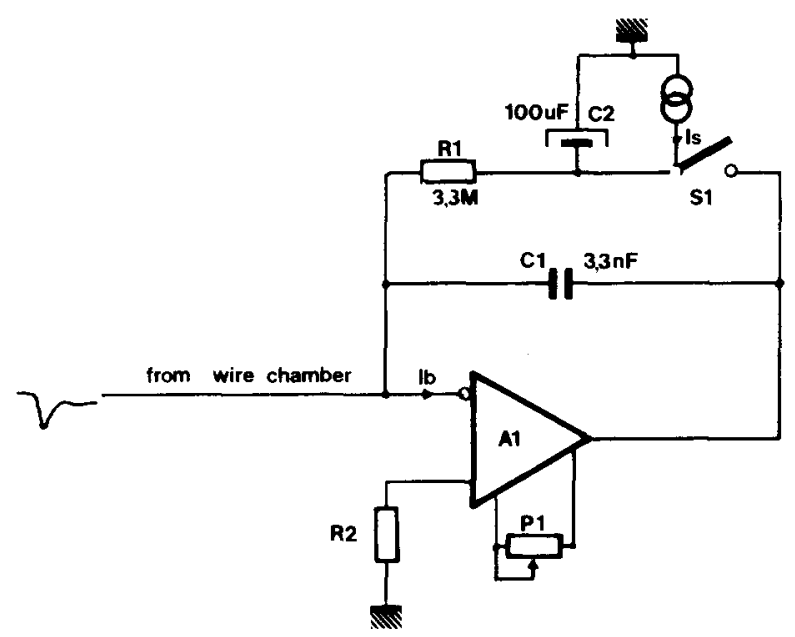

Fig. 3. Electrical circuit diagram of a drift compensated charge integrator.

tegrator must be of $1 \mathrm{mV}$. These values impose a maximum leakage current

$I_{\mathrm{L}}=\frac{C_{1} \Delta V}{\Delta t} \leq 2 \mathrm{pA}$,

where $\Delta t=$ integration time $(1.4 \mathrm{~s})$.

A scheme of the charge integrator is given in fig. 3. The amplifier is a low cost FET operational amplifier $\left(A_{1}\right)$ with an input bias current $I_{\mathrm{B}} \leq 50 \mathrm{pA}$ and the switch $\left(\mathrm{S}_{1}\right)$, which is used to discharge $C_{1}$, is a current CMOS circuit with a leakage current $I_{\mathrm{S}} \leq 1000 \mathrm{pA} \quad(10 \mathrm{pA}$ typical). To bring the total leakage current at $\leq 2 \mathrm{pA}$, an automatic compensation has been introduced. It consists of a storing capacitor $\left(C_{2}\right)$ and $R_{1}$. Outside the integration time, $S_{1}$ is closed and $A_{1}$ looks like a unity inverting amplifier, where the output voltage is adjusted to zero by $P_{1}$. The total leakage current $I_{\mathrm{L}}=I_{\mathrm{S}}+I_{\mathrm{B}}$ is now furnished by the $\mathrm{A}_{1}$ output.

When $S_{1}$ is opened (integrator mode), $I_{L}$ is furnished by $C_{2}$ and the output voltage drift is now

$\Delta v_{\text {out }}=\frac{I_{\mathrm{L}}}{C_{1}}\left[\Delta t-R_{1} C_{2}\left(1-\mathrm{e}^{-\Delta t / R_{1} C_{2}}\right)\right]$.

For $R_{1} C_{2} \gg \Delta t$ a substantial improvement is obtained. In practice for $\Delta t=1.4 \mathrm{~s}, R_{1}=3.3 \times 10^{6} \Omega$, $C_{2}=100 \mu \mathrm{F}, \Delta v_{\text {out }}$ becomes 500 times smaller than whithout correction and allows us to use low cost components.

We wish to thank Dr. A. Placci (who is at the origin of this project) and Mr. M. Rabany for useful discussions and suggestions, $\mathrm{Mr}$. C. Guillaume for his assistance in the use of the CAMAC modules and Dr. B. Skaali who designed the software for computer acquisition and display.

\section{Reference}

1) C. Guillaume, CERN internal report SPS/CO/Int/CC/76-3. 